\title{
DEFORMATIONS OF A TWO-GENERATOR PURELY INSEPARABLE FIELD OF EXPONENT 1
}

\author{
FRANCIS P. CALLAHAN
}

\begin{abstract}
This paper gives a discussion of the algebraic deformations of a two-generator purely inseparable field of exponent 1 , this being the simplest field having noncommutative deformations. It gives a necessary condition for a 2-cocycle to be integrable and, if it is allowed to adjoin a single separable element to the groundfield, also a sufficient condition for a 2-cocycle to be integrable.

The method used is to study certain skew symmetric biderivations associated with the field.
\end{abstract}

Introduction. This paper considers algebraic deformations [1] of a two-generator purely inseparable field of exponent 1, [2], this being the simplest field having noncommutative deformations. (In what follows, "field" means "twogenerator purely inseparable field of exponent 1".) As shown in [1] what may be called the generator or infinitesimal of a deformation is always a 2-cocycle in the sense of [3], although not every 2-cocycle is the infinitesimal of a deformation-in-the-large; the question of whether or not a 2-cocycle is the infinitesimal of a deformation depends only on its cohomology class as is also shown in [1], so that we may speak of the cohomology class as being integrable or not integrable. Here we give a necessary condition for integrability which is also sufficient if it is permitted to adjoin a single separable element to the groundfield. Whether it is in fact necessary to adjoin this element to the groundfield is not known for general characteristic $p$, but for $p=2$, it is trivially not necessary.

The method used in this paper is first to study certain skew symmetric biderivations defined on the field being considered so as to show that these biderivations can be put into a convenient form by proper choice of generators for the field. (Finding these generators may entail adjoining a separable element to the groundfield.)

Then this theorem is applied to deformation theory for the field so as to characterize its deformations.

1. Skew symmetric biderivations. We begin with some preliminaries about the field being considered. Let $A$ be a field of dimension $p^{2}$ over $k$, the groundfield ( $\operatorname{char} k=p \neq 0$ ) and let there be no $x$ in $A$ such that the subalgebra of $A$ generated by $x$ is all of $A$. Then it is easy to show that any $x$

Received by the editors March 25, 1975 and, in revised form, January 5, 1976.

AMS (MOS) subject classifications (1970). Primary 13B10, 13D10.

() American Mathematical Suciety 1977 
in $A$ satisfies an equation of the form $x^{p}=X$ where $X$ is in $k$. Such a field is called a purely inseparable field of exponent 1 . (In general, a purely inseparable field is one in which each element satisfies an irreducible purely inseparable equation of the form $x^{m}=X$ where $X$ is in $k$, and $m=p^{n}$.) (The exponent of the field is the supremum of $n$ over all elements in the field. See [2].) If $y$ is a second element of $A$ not in the subalgebra generated by $x$, then any $u$ in $A$ can be written uniquely as $u=\sum u_{i j} x^{i} y^{j}(0 \leqslant i, j \leqslant p-1)$. Then $x$ and $y$ are called a generator pair for the field.

"Derivation" is defined as usual and a skew symmetric biderivation is a $k$-linear mapping, $\phi$, from $A \times A$ to some two sided $A$-module (in the present case, $A$ itself) such that the mapping is a derivation in each of its arguments and such that $\phi(u, u)=0$ for all $u$.

Proposition 1. Let $(x, y)$ be a generator pair for field $A$ and let $\phi$ be a skew symmetric biderivation on $A$. Then, for $u$, $v$ in $A$,

$$
\phi(u, v)=\left(u_{x} v_{y}-v_{x} u_{y}\right) \phi(x, y) \text {. }
$$

Here $u_{x}$ is an abbreviation for $D_{x}(u)$ where $D_{x}$ is the standard derivation defined by $D_{x}(x)=1$ and $D_{x}(y)=0 \cdot u_{y}, v_{x}$ and $v_{y}$ are defined similarly. Conversely, any choice of $\phi(x, y)$ in (1) defines a skew symmetric biderivation.

Proof. Note first that for any $u$ in $A$, and for any derivation, $D, D(u)=$ $u_{x} D(x)+u_{y} D(y)$ (easily established for $u=x^{i} y^{j}$ and extended to general $u$ by $k$-linearity).

Thus,

$$
\phi(u, v)=u_{x} \phi(x, v)+u_{y} \phi(y, v)
$$

since $\phi$ is a derivation in its first argument.

Further,

$$
\begin{gathered}
\phi(x, v)=v_{x} \phi(x, x)+v_{y} \phi(x, y)=v_{y} \phi(x, y), \\
\phi(y, v)=v_{x} \phi(y, x)=-v_{x} \phi(x, y) .
\end{gathered}
$$

(We are using here $\phi(x, y)+\phi(y, x)=0$, which follows by a well-known argument from $\phi(u, u)=0$.) The result now follows by substitution.

The formula for the biderivation becomes particularly simple when $\phi(x, y)$ $=1: \phi(u, v)=u_{x} v_{y}-u_{y} v_{x}$. This leads us to ask under what circumstances $A$ has a generator pair, $(r, s)$ for which $\phi(r, s)=1$ for a given $\phi$. Such a skew symmetric biderivation will be called a "special" skew symmetric biderivation.

Proposition 2, just below, gives a necessary condition for a skew symmetric biderivation to be special. Theorem 1 in this section, gives a sufficient condition.

Proposition 2. Let $\phi$ be a skew symmetric biderivation on $A$ and let $(r, s)$ be such that $\phi(r, s)=1$. Let $(x, y)$ be another generator pair for $A$ and let $1 / \phi(x, y)=f=\sum f_{i j} x^{i} y^{j}\left(0 \leqslant i, j \leqslant p-1\right.$ and $f_{i j}$ in $\left.k\right)$. Then $f_{p-1 \cdot p-1}=0$. 
Proof. $1=\phi(r, s)=\left(r_{x} s_{y}-r_{y} s_{x}\right) \phi(x, y)$ (by equation (1)) so that $f=$ $1 / \phi(x, y)=r_{x} s_{y}-r_{y} s_{x}$. Now, with the usual conditions on the summations, let $r=\sum r_{i j} x^{i} y^{j}$ and $s=\sum s_{i j} x^{i} y^{j}$. Then

$$
r_{x} s_{y}-r_{y} s_{x}=\sum \sum r_{i j} s_{m n}(i n-j m) x^{i+m+1} y^{j+n+1} .
$$

Thus the coefficient of $x^{p-1} y^{p-1}$ in $f$ is $\Sigma \sum r_{i j} s_{m n}(i n-j m)$ where the summation is over such values that $i+m-1 \equiv p-1(\bmod p)$ and $j+n-1 \equiv p$ $-1(\bmod p)$. This causes $i n-j m$ to vanish $(\bmod p)$ and gives the result.

From Proposition 2 we see that the vanishing of the coefficient of $x^{p-1} y^{p-1}$ in $1 / \phi(x, y)$ is a necessary condition for the existence of a generator pair in $A$ for which $\phi(r, s)=1$. Whether it is a sufficient condition for such a pair to exist in $A$ is not known; however, the main theorem shows that it is a sufficient condition for the existence of such a pair in an extension of $A$ gotten by adjoining at most one element to the groundfield, $k$.

THEOREM 1. Let $A$ be a field of the type described above and let $\phi$ be a skew symmetric biderivation on it. Let the coefficient of $x^{p-1} y^{p-1}$ in $1 / \phi(x, y)$ be zero. Then $A$ can always be extended by adjoining at most a single element to $k$, the groundfield, in such a way that the extension contains a pair $(r, s)$ for which $\phi(r, s)=1$.

Before giving the proof we give a simple lemma.

LEMMA 1. Let $\phi$ be as in the theorem. Then either

(1) there is a generator pair, $(r, s)$, in $A$ such that $\phi(r, s)=1$, or

(2) there is a pair for which $1 / \phi(r, s)=s^{p-1}+\sum g_{j} s^{j}$ (in the summation $j$ runs from 0 to $p-2$ and each $g_{j}$ is a polynomial in $r$ with coefficients in $k$ ).

Proof. Let $1 / \phi(x, y)=\sum f_{i j} x^{i} y^{j}$ and let $f_{j}=\sum f_{i j} x^{i}$, so that $1 / \phi(x, y)=$ $\sum f_{j} y^{j}$. (Limits on the summations should be obvious at this point.) Suppose first that $f_{p-1}=0$. Then there is an $s$ such that $s_{y}=1 / \phi(x, y)$. Letting $r=x$ then gives easily $\phi(r, s)=1$. This is case (1).

Suppose next that $f_{p-1} \neq 0$, and note that $f_{p-1}$ cannot contain a term in $x^{p-1}$, for if it did, $1 / \phi(x, y)$ would contain a term in $x^{p-1} y^{p-1}$. Thus there is an $r$ such that $r_{x}=f_{p-1}$ and $r_{y}=0$. Letting $y=s$ now leads by a simple computation to the form for $\phi(r, s)$ given in case (2).

As a result of the lemma we need only consider the case in which $1 / \phi(x, y)=y^{p-1}+\sum f_{j} y^{j}, f_{j}$ being a polynomial in $x$ and the summation going from 0 to $p-2$. (We have replaced the $r, s$ of the lemma by $x, y$ for convenience.)

Proof of THEOREM. If $A$ contains no generator pair such that $\phi(r, s)=1$, select a pair $(x, y)$ such that $1 / \phi(x, y)=y^{p-1}+\sum f_{j} y^{j}$ as in the lemma.

Now consider $x$ to be fixed and define a derivation, $\theta(v)$, by $\theta(v)=$ $\phi(x, v)$. Since $\phi(x, x)=0, \theta(x)=0$, and we can take $x$ to be in the groundfield as far as $\theta$ is concerned. That is, view $A$ as $k^{\prime}(y)$ where groundfield $k^{\prime}$ is $k(x)$; then $\theta$ is a derivation on $A$ viewed this way. Next we apply the results of [2]. It is shown there that if $k^{\prime}(y)$ is a purely inseparable field gotten by 
adjoining a $y$ such that $y^{p}=Y$ ( $Y$ in $k^{\prime}$ and the equation irreducible) to $k^{\prime}$ and if $\theta$ is a derivation on $k^{\prime}(y)$ (so that there is a $u$ for which $\theta=$ $(1 / u) d / d y)$, then $\theta$, viewed as a linear transformation, has characteristic equation $\lambda^{p}=b \lambda$ where $b=-(d / d y)^{p-1}(u) / u^{p}$.

Thus, after adjoining, if necessary, a root of $\lambda^{p-1}=b$ to $k^{\prime}$, we can find an eigenvector, $e$, such that $\theta(e)=\lambda e$. In the present case, because of the form of $\phi(x, y), u=y^{p-1}+\sum f_{j} y^{j}$, and $-(d / d y)^{p-1}(u)=1$, so that $b=$ $1 /\left(Y^{p-1}+\sum f_{j}^{p} Y^{j}\right)$. This shows that $b$ is not only in $k^{\prime}$ but actually in $k$, the original groundfield for $A$, so that $\lambda$ need only be adjoined to $k$. This allows the extension of the definition of the biderivation $\phi$ to $A^{\prime}$, which is $A$ with $\lambda$ adjoined to $k$; if $\lambda$ had to be adjoined to $k^{\prime}$, difficulties would arise in counting $\lambda$ as part of the groundfield of $A^{\prime}$ because $\lambda$ would be related to $x$.

Finally, since $\phi(x, e)=\lambda e$ and since $\phi(e, e)=0$, and $\phi(x / \lambda \dot{e}, e)=1$, the desired pair $(r, s)$ can be taken to be $(x / \lambda e, e)$.

COROLlaRY If biderivation $\phi$ is special with respect to one basis then it is special with respect to every basis.

Proof. If $\phi$ is special with respect to one basis then there exists a generator pair $(r, s)$ such that $\phi(r, s)=1$. Then, because of Proposition 2, $\phi$ is special with respect to every generator pair.

Corollary. A skew symmetric biderivation is special if and only if there exist polynomials $f$ and $g$ (in $x$ and $y$ ) such that $D_{x} f+D_{y} g=1 / \phi(x, y)$. $f$ and $g$ are not unique.

Proof. This follows easily from the fact that $\phi$ is special if and only if $1 / \phi(x, y)$ does not contain a term in $(x y)^{p-1}$.

2. Application to deformations. The field $A$ is still a two-generator purely inseparable field of exponent 1 . Now we consider noncommutative deformations of $A$.

Note first that if $A_{t}$ is a noncommutative deformation of $A$ then any $x$ in $A_{t}$ but not in $k_{t}$, the ground ring, satisfies an irreducible equation of degree $p$. To see this observe that $x$ does satisfy some irreducible equation and that the degree of this equation must divide $p^{2}$, the dimension of $A_{t}$ over $k_{t}$, as is easy to show. The degree cannot be $p^{2}$ because then $A_{t}$ would have a single generator and thus be commutative; hence the degree is $p$.

Thus, let

$$
k_{0}+k_{1} x \cdots+k_{p} x^{p}=0
$$

where each $k_{i}$ is in $k_{t}$. If $x=x_{0}+t x_{1}+t^{2} x_{2}+\ldots$ where each $x_{i}$ is in $A$, then (2) reduces to the equation (in $A$ ) for $x_{0}$ when we set $t=0$. Letting this be $x_{0}^{p}=X_{0}$, we see that $k_{p}=1+t k_{p_{1}}+\ldots$. From this it is easy to see that $k_{p}$ has an inverse in ground ring $k_{t}$. Thus, multiplying equation (2) by this inverse we see that $k_{p}$ can be taken to be 1 without real loss of generality. It 
follows also that each $k_{i}$ is divisible by $t$.

Thus, (2) can be rewritten as

$$
x^{p}+f(x)=0
$$

where $f$ is a polynomial in $x$ of degree at most $p-1$ with coefficients in $k_{t}$ and having each coefficient (except the 0th term) divisible by $t$.

Now we prove the following

Identity. Let $x$ be a member of an associative ring of characteristic $p$ and let $f$ be a polynomial of degree not greater than $p-1$ in $x$, and let primes designate differentiation by $x$.

Then ad $f(x)=f^{\prime} \operatorname{ad}(x)-\frac{1}{2} f^{\prime \prime}(\operatorname{ad} x)^{2}+\ldots-f^{(p-1)}(\operatorname{ad} x)^{p-1} /(p-1) !$ as usual, ad $f$ is defined by

$$
\operatorname{ad} f(y)=f y-y f=f_{L} y-f_{R} y .
$$

(Here $f_{L}$ means multiplication on the left by $f$ and $f_{R}$ means multiplication on the right.)

Thus, $\operatorname{ad}(f)=f_{L}-f_{R}$ and $f_{R}=f_{L}-\operatorname{ad}(f)$. Now take $f=x^{i}$. Then,

$$
\left(x_{R}\right)^{i}=\left(x_{L}-\operatorname{ad}(x)\right)^{i}=\sum_{j=0}^{i}\left(\begin{array}{l}
i \\
j
\end{array}\right) x_{L}^{i-j}(-\operatorname{ad}(x))^{j}
$$

so that

$$
x_{L}^{i}-x_{R}^{i}=-\sum_{j=1}^{i}\left(\begin{array}{c}
i \\
j
\end{array}\right) x_{L}^{i-j}(-\operatorname{ad}(x))^{j}
$$

From this the identity follows easily for $f=x^{i}$ and by superposition for the general $f$. For $i=p$ we obtain the well-known identity $\operatorname{ad}\left(x^{p}\right)=(\operatorname{ad}(x))^{p}$. More generally, the identity is still true if $f$ is a polynomial in $x$ and $y$ provided that differentiation is partial, $D_{x}$.

One kind of noncommutative deformation of such a field is described in [4]. The multiplication law is given by

$$
u v=\sum \frac{t^{n i}}{i !} D_{x}^{i}(u) D_{y}^{i}(v) \quad(0 \leqslant i \leqslant p-1) .
$$

$D_{x}$ and $D_{y}$ are the standard derivations previously described. We show now that all noncommutative deformations of $A$ are essentially of the type described in (1) provided an appropriate generator pair is chosen.

THEOREM 2. (Necessity.) Let a deformation of the type specified in (1) be given. Then the associated cocycle is a skew symmetric biderivation; furthermore, it is special.

Proof. Let $u v-v u=[u, v]=\operatorname{ad} u(v)$. Then it is well known that $[u, v]$ is a skew symmetric biderivation on $A_{t}$. If we write

$$
[u, v]=t \phi_{1}(u, v)+t^{2} \phi_{2}(u, v) \ldots
$$

then $\phi_{1}$ can be viewed as a mapping from $A \times A$ to $A$ and is in fact the cocycle associated with the deformation. It is skew symmetric, a biderivation 
and special (because $[x, y]=1$ ) all of which is easy to verify.

Before proving the next theorem we prove two lemmas.

LEMMA 2. Let $A_{t}$ be a noncommutative deformation of $A$ and define $a$ sequence of skew symmetric biderivations, $\phi, \phi_{1}, \ldots$ from $A \times A$ to $A$ by means of the formula

$$
r s-s r=t^{\alpha}\left(\phi(r, s)+t \phi_{1}(r, s)+\ldots\right) .
$$

Then $\phi$ is special.

Proof. Let $(x, y)$ generate $A_{t}$ and let $x^{p}+f(x)=0$ so that $f$ satisfies the Identity proven above. Let $U$ be the operator from $A$ to $A$ defined by $U(z)=-\phi(x, y) D_{y}(z)$. Then it is easily shown that $(\operatorname{ad}(x))^{i}(y)=\left(t^{\alpha} U\right)^{i}(y)$ $+\cdots+$.

Let $f^{(i)}=t^{n_{i}} g_{i}$ where $f^{(i)}$ is the $i$ th derivative of $f$ and $g_{i}$ is a polynomial in $x$ not divisible by $t$. It is easy to see that $n_{1}, n_{2}, \ldots$, is a nondecreasing sequence of integers.

Thus, the Identity becomes in this case

$$
t^{n_{1}+\alpha} g_{1} U(y)-\frac{1}{2} t^{n_{2}+2 \alpha} g_{2} U^{2}(y)+\cdots+t^{p \alpha} U^{p}(y)+\ldots=0 .
$$

Now clearly the first term can only cancel with the last because the powers of $t$ in the intervening terms are too high. Thus, $n_{1}=(p-1) \alpha$ and $g_{1} U(y)+$ $U^{p}(y)=0$. Now, as in [2] it can be shown that $U^{p}=b U$ where $b=$ $(\phi(x, y))^{p} D_{y}^{p-1}(1 / \phi(x, y))$. Since $f^{\prime}=t^{n_{1}} g_{1}$ it follows that $g_{1}$ is a polynomial of degree not higher than $p-2$ in $x$ and since

$$
g_{1}+\phi(x, y)^{p} D_{x}^{p-1}\left(\frac{1}{\phi(x, y)}\right)=0
$$

it now follows easily that $(x y)^{p-1}$ is missing from $1 / \phi(x, y)$, so that $\phi$ is special.

Thus, without real loss of generality we can choose a basis $(x, y)$ such that

$$
x y-y x=t^{\alpha}+t^{\beta} \phi_{1}(x, y)+\ldots
$$

LEMMA 3. The mapping $\phi_{1}$ in equation (5) is a special skew symmetric biderivation.

Proof. We perform a computation similar to that of Lemma 2 taking into account the next terms in the series:

$$
(\operatorname{ad}(x))^{i}(y)=t^{\beta}\left(-t^{\alpha} D_{x}\right)^{i-1} \phi_{1}(x, y)+\ldots \quad \text { for } i>1
$$

for $i=1 \operatorname{ad}(x)(y)$ is given by (5). Again we obtain an equation similar to (4):

$$
g_{1} \phi_{1}(x, y)+D_{x}^{p-1} \phi_{1}(x, y)=0 \text {. }
$$

This shows that $1 / \phi_{1}(x, y)$ is a polynomial in $x$ only and this shows that $\phi_{1}$ is special. Of course, the same argument would show that $\phi_{1}(x, y)$ involves $y$ only, so that in fact, $\phi_{1}(x, y)$ must be in $k_{t}$, but this is more than we need. 
THEOREM 3. Given a noncommutative deformation, $A_{t}$, of $A$ a single separable element can always be adjoined to $k$ such that the resulting extension, $A_{t}^{\prime}$, of $A_{t}$, always contains a generator pair $(r, s)$ such that $[r, s]=t^{\alpha}$.

Proof. Because of Lemmas 2 and 3 we can find generators $r$ and $s$ for $A_{t}$ such that

$$
r s-s r=t^{\alpha}+t^{\beta} \phi_{1}(r, s)
$$

where $\phi_{1}$ is special. Because $\phi_{1}$ is special there exist polynomials $f$ and $g$ in $x$ and $y$ such that $\phi_{1}(x, y)=-D_{x} f+D_{y} g$. Put $r^{\prime}=r+t^{\beta} f$ and $s^{\prime}=s+t^{\beta} g$.

Then $r^{\prime} s^{\prime}-s^{\prime} r^{\prime}=t^{\alpha}+t^{\gamma} \phi_{2}\left(r^{\prime}, s^{\prime}\right)$ where $\gamma>\beta$ and $\phi_{2}$ is again special by Lemma 3. A sequence of such transformations serves to produce the desired basis in the limit.

To complete the discussion it remains to show that the generators $r$ and $s$ are such that $r^{p}$ and $s^{p}$ are in the extended ground ring $k_{t}^{\prime}$. To do this note that $(\operatorname{ad}(r))^{2}(s)=0$, so that $0=(\operatorname{ad}(r))^{p}(s)=\operatorname{ad}\left(r^{p}\right)(s)$. Thus $r^{p}$ commutes with $s$; since it also commutes with $r$ it is in $k_{t}^{\prime}$; similarly for $s^{p}$.

ACKNOWLEDGEMENT. Sincere thanks are hereby given to the referee for his helpful comments.

\section{REFERENCES}

1. M. Gerstenhaber, On the deformation of rings and algebras, Ann. of Math. (2) 79 (1964), 59-103. MR 30 \#2034.

2. N. Jacobson, Lectures in abstract algebra. Vol 3: Theory of fields and Galois theory, Van Nostrand, Princeton, N.J., 1964, p. 19, Exercise 15. MR 30 \#3087. Or, in a different context:

$2^{\prime}$. F. Callahan, An identity satisfied by derivations of a purely inseparable field, Amer. Math. Monthly 80 (1973), 40-42.

3. G. Hochschild, On the cohomology groups of an associative algebra, Ann. of Math. (2) 46 (1945), 58-67. MR 6, 114.

4. M. Gerstenhaber, On the deformation of rings and algebras. III, Ann. of Math. (2) 83 (1968), 1-34. MR 39 \# 1521.

King of Prussia Graduate Center, Pennsylvania State University, King of Prussia, Pennsylvania 19406 